\title{
Value of fusion of PET and MRI in the detection of intra-pelvic recurrence of gynecological tumor: comparison with ${ }^{18}$ F-FDG contrast-enhanced PET/CT and pelvic MRI
}

\author{
Kazuhiro Kitajima Yuko Suenaga Y Yoshiko Ueno • Tomonori Kanda • \\ Tetsuo Maeda $\cdot$ Natsuko Makihara $\cdot$ Yasuhiko Ebina $\cdot$ Hideto Yamada \\ Satoru Takahashi $\cdot$ Kazuro Sugimura
}

Received: 17 September 2013/Accepted: 3 October 2013/Published online: 16 October 2013

(C) The Japanese Society of Nuclear Medicine 2013

\begin{abstract}
Background To evaluate the diagnostic value of retrospective image fusion from pelvic magnetic resonance imaging (MRI) and ${ }^{18} \mathrm{~F}$-fluorodeoxyglucose positron emission tomography (PET) in detecting intra-pelvic recurrence of gynecological tumor.

Methods Thirty patients with a suspicion of recurrence of gynecological malignancy underwent inline contrastenhanced PET/computed tomography (CT) and pelvic contrast-enhanced MRI for restaging. Diagnostic performance about the local recurrence, pelvic lymph node and bone metastasis and peritoneal lesion of PET/low-dose non-enhanced CT (PET/ldCT), PET/full-dose contrastenhanced CT (PET/ceCT), contrast-enhanced MRI, and retrospective image fusion from PET and MRI (fused PET/ MRI) were evaluated by two experienced readers. Final diagnoses were obtained by histopathological examinations, radiological imaging and clinical follow-up for at
\end{abstract}

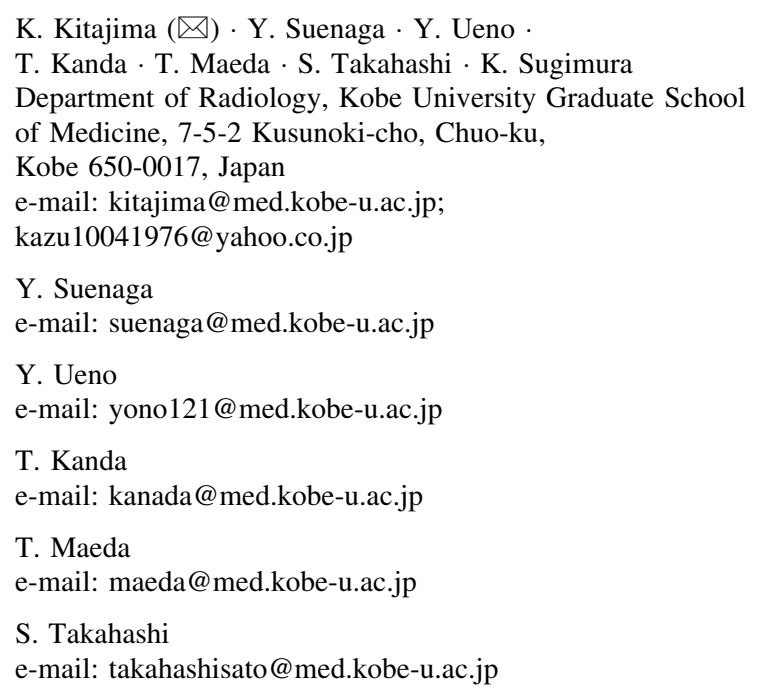

least 6 months. McNemar test was employed for statistical analysis.

Results Documented positive locally recurrent disease, pelvic lymph node and bone metastases, and peritoneal dissemination were present in 53.3, 26.7, 10.0, and $16.7 \%$, respectively. Patient-based sensitivity for detecting local recurrence, pelvic lymph node and bone metastasis and peritoneal lesion were $87.5,87.5,100$ and $80.0 \%$, respectively, for fused PET/MRI, 87.5, 62.5, 66.7 and $60.0 \%$, respectively, for contrast-enhanced MRI, 62.5, 87.5, 66.7 and $80.0 \%$, respectively, for PET/ceCT, and 50.0, 87.5, 66.7 and $60.0 \%$, respectively, for PET/ldCT. The sensitivity of diagnosing local recurrence by fused PET/MRI was significantly better than that of PET/ldCT $(p=0.041)$. The patient-based sensitivity, specificity and accuracy for the detection of intra-pelvic recurrence/ metastasis were $91.3,100$ and $93.3 \%$ for fused PET/MRI, 82.6, 100 and $86.7 \%$ for contrast-enhanced MRI, 82.6, 100

\author{
K. Sugimura \\ e-mail: sugimura@med.kobe-u.ac.jp \\ K. Kitajima $\cdot$ Y. Suenaga $\cdot$ Y. Ueno - T. Kanda - T. Maeda $\cdot$ \\ N. Makihara $\cdot$ Y. Ebina $\cdot$ H. Yamada $\cdot$ S. Takahashi · \\ K. Sugimura \\ Department of Radiology, Hyogo Cancer Center, Hyogo, Japan \\ e-mail: makiharana@med.kobe-u.ac.jp \\ Y. Ebina \\ e-mail: ebinaa@med.kobe-u.ac.jp \\ H. Yamada \\ e-mail: yamada-hide@med.kobe-u.ac.jp \\ N. Makihara $\cdot$ Y. Ebina $\cdot$ H. Yamada \\ Department of Obstetrics and Gynecology, Kobe University \\ Graduate School of Medicine, 7-5-2 Kusunoki-cho, Chuo-ku, \\ Kobe 650-0017, Japan
}


and $86.7 \%$ for PET/ceCT and 78.3, 85.7 and $80.0 \%$ for PET/ldCT.

Conclusion Fused PET/MRI combines the individual advantages of MRI and PET, and is a valuable technique for assessment of intra-pelvic recurrence of gynecological cancers.

Keywords Fused PET/MRI - PET/CT · MRI ·

Restaging · Gynecological tumor

\section{Introduction}

Inline positron emission tomography/computed tomography (PET/CT), a combination of PET and CT with ${ }^{18} \mathrm{~F}$ fluorodeoxyglucose $\left({ }^{18} \mathrm{~F}-\mathrm{FDG}\right)$, is now accepted as a powerful imaging modality in evaluating various kinds of malignancies including gynecological cancers $[1,2]$. PET/ CT has been reported to be more useful than PET alone, with helpful anatomical and morphological information from its CT portion.

However, contrast resolution of CT for different tissues is limited especially in the pelvis and head and neck even when full-dose exposure and contrast medium are employed. In contrast, magnetic resonance imaging (MRI) has several advantages over $\mathrm{CT}$, such as high tissue contrast and no radiation exposure. Because of the higher tissue contrast by MRI in gynecological cancers than with $\mathrm{CT}$, fusion of MR and PET images (fused PET/MRI) may result in some clinical advantages for gynecological cancers over PET/CT. However, the clinical application of fused PET/MRI of the pelvis in gynecological cancer patients is still limited [3-5].

Moreover, although inline whole-body PET/MRI has been developed recently, it remains unknown whether the device is clinically applicable. To begin with, the clinical value of image fusion from MRI and PET in the gynecological disease needs to be clarified. The purpose of this study was to evaluate the validity of retrospectively fused PET/MRI obtained from different scanners for assessing intra-pelvic recurrence of gynecological cancers and to compare the diagnostic accuracy of fused PET/MRI with that of inline PET/full-dose contrast-enhanced CT (PET/ ceCT) and PET/low-dose non-enhanced CT (PET/ldCT) with ${ }^{18} \mathrm{~F}$-FDG, and contrast-enhanced MRI.

\section{Materials and methods}

\section{Patients}

This retrospective study was approved by the institutional review board in our institute, and the need for patient informed consent was waived. From December 2011 to January 2013, thirty patients (mean age 61.3 years; age range 38-83 years) previously treated for uterine cervical cancer $(n=15)$, ovarian cancer $(n=9)$ and endometrial cancer $(n=6)$ underwent conventional ${ }^{18} \mathrm{~F}$-FDG PET/CT scan with low-dose CT (ldCT) followed by full-dose with IV contrast (ceCT) and pelvic contrast-enhanced MRI with a suspicion of recurrence including elevated tumor marker levels, abnormal CT or MRI findings, or an abnormal Papanicolaou smear. All patients received the histopathological result by biopsy or surgery, or clinical follow-up including PET/CT, $\mathrm{CT}$, and MRI examinations for longer than 6 months. The maximum interval between MRI and PET/CT acquisition was 21 days (mean 7.7 days; range 0-21 days).

\section{Pelvic MRI}

All subjects were examined using a $1.5 \mathrm{~T}$ MR scanner (Achieva 1.5 T, Philips Medical Systems, Best, The Netherland) with a pelvic phased-array coil. No intramuscular injection of butyl scopolamine or glucagon was used.

Unenhanced T1-weighted images (T1WIs) were acquired in the axial and sagittal planes with a spin-echo repetition time (TR)/echo time (TE) $=525-702 /$ 5.5-9.8 ms sequence, $4-5 \mathrm{~mm}$ slice thickness $/ 1 \mathrm{~mm}$ gap, a $20-24 \mathrm{~cm}$ field of view, and a $192 \times 256$ matrix. Axial, sagittal and coronal T2-weighted fast-spin-echo images (T2WIs); 3,500-4,600/90 ms, 4-5 mm slice thickness/ $1 \mathrm{~mm}$ gap, a $20-24 \mathrm{~cm}$ field of view, and a $192 \times 256$ matrix) were obtained. Axial diffusion weighted imaging (DWI) with $b$ value of 0 and $1,000 \mathrm{~ms}$ were also obtained. After administration of $0.08-0.1 \mathrm{mmol} / \mathrm{kg}$ of gadolinium diethylenetriaminepentaacetic acid (DTPA) (Magnevist: Bayer Schering Pharma, Osaka, Japan), we obtained T1weighted fat-suppressed axial, sagittal, and coronal sequences sequentially, with parameters similar to those used before the gadolinium DTPA injection.

\section{${ }^{18}$ F-FDG contrast-enhanced PET/CT (PET/ceCT)}

Whole-body imaging was performed using a combined PET/CT scanner (Discovery PET/CT 690, GE Healthcare, Waukesha, WI, USA). CT covered a region ranging from the meatus of the ear to the midthigh. The technical parameters of the 16-detector row helical CT scanner were a helical pitch of 28 or a beam pitch of 1.75 , a gantry rotation speed of $0.6 \mathrm{~s}$, and a slice thickness of $3.27 \mathrm{~mm}$. The PET component of the combined imaging system allows simultaneous acquisition of 47 transaxial PET images with an interslice spacing of $3.27 \mathrm{~mm}$ in one bed position and provided an image from the meatus of the ear to the midthigh with $7-8$ bed positions. The transaxial field of view and axial field of view of the PET images 
reconstructed for fusion were 60 and $15.0 \mathrm{~cm}$, respectively, with a matrix size of $192 \times 192$. To avoid artifacts caused by the urinary tract, patients were asked to drink $500 \mathrm{ml}$ of water $1-2 \mathrm{~h}$ prior to image acquisition, and to void just before the start of acquisition. No urinary bladder catheterization was used. After at least $4 \mathrm{~h}$ of fasting, patients received an intravenous injection of $222-333 \mathrm{MBq}$ $(6-9 \mathrm{mCi})$ of ${ }^{18} \mathrm{~F}-\mathrm{FDG}$. The blood glucose levels were checked in all patients before FDG injection and no patients showed a blood glucose level of more than $160 \mathrm{mg} / \mathrm{dL}$.

About 50 min later, initially ldCT was performed at $120 \mathrm{kV}$ and Smart mA (20-120 mA, Noise Index 30) with the normal expiration position for attenuation correction of PET image. A whole-body emission PET scan was performed immediately after the $1 \mathrm{dCT}$, with a 2-min acquisition per bed position using a three-dimensional acquisition mode. Attenuation-corrected PET images were reconstructed with an ordered-subset expectation maximization iterative reconstruction algorithm called VUE Point FX-S with TOF and sharp IR (18 subsets, 2 iterations).

Finally, diagnostic ceCT was performed for the same axial coverage at $140 \mathrm{kV}$ and Auto $\mathrm{mA}(20-300 \mathrm{~mA}$, Noise Index 10) and $35.0 \mathrm{~mm} /$ rotation speed, during breath hold with the normal expiration positions, similar to ldCT scanning. Iodinated contrast material (Iopamiron Inj, Syringe, Bayer Schering Pharma, Berlin, Germany) containing 300 or $370 \mathrm{mg}$ of iodine per milliliter at a dose of $450 \mathrm{mg}$ of iodine per kilogram of body weight was intravenously administered by using a power injector with a fixed injection duration of $50 \mathrm{~s}$. The scan of neck-thorax and abdomen-pelvis was started at 50 and $100 \mathrm{~s}$, respectively, after injection. No oral contrast agent was administered. For image fusion, $3.27 \mathrm{~mm}$ slice was reconstructed. The $\mathrm{ldCT}$, ceCT, and PET images were transferred to a commercially available workstation (Advantage Windows Workstation, version 4.5, GE Healthcare Technology) to access all data (Fig. 1).

\section{Fused PET/MRI}

PET and all MRI series (T1WI, T2WI and contrastenhanced imaging) were retrospectively and manually fused using a dedicated, commercially available software (Advantage Windows Workstation, version 4.5, GE Healthcare Technology). This automatic rigid fusion software has already been used in other studies for image fusion of PET and MRI [3, 5, 6]. MR and PET/CT images were stored in a common database and the MR images were registered to CT images of PET/CT using a semiautomatic voxel-based algorithm. After registration, the coregistered images were reconstructed and visualized in the axial planes. Alignment in all three planes (axial, coronal, and sagittal) was assessed by checking the body outline and the position of metabolically active motionless organs (bone and spine). When the accurate image fusion was not feasible, PET-MRI fusion was evaluated by assessing PET/MRI-fused images side by side with PET and MRI images.

Image analysis

Images were analyzed on a dedicated workstation (Advantage Windows, version 4.5; GE Healthcare Europe). Two board-certificated radiologists/nuclear medicine physicians (both having double board-certifications) who are especially experienced in gynecology imaging, consensually and retrospectively evaluated MRI, PET/ceCT, PET/ ldCT, and fused PET/MRI for the following findings: (a) local pelvic recurrence, (b) pelvic lymph node metastasis, (c) pelvic bone metastasis, and (d) peritoneal dissemination in consensus. Neither reader was aware of the results of other imaging studies, histopathologic findings or the clinical data. Each dataset was reviewed with the consensus of the two readers after a minimum interval of 3 weeks to avoid any decision threshold bias due to reading-order effects.

We referred several previous standard criteria about restaging gynecological cancer on MRI [7-9] and PET/CT interpretation $[10,11]$. On MRI interpretation, lymph node swelling larger than $1 \mathrm{~cm}$ in short-axis diameter was graded as metastasis. On PET/CT and fused PET/MR interpretations, the classification of LNs as cancer positive was based on the presence of focally appreciable metabolic activity above that of comparable normal structures or surrounding tissue, with the exclusion of physiological bowel, vessel, and urinary activity [12]. Furthermore, the presence of a central unenhancing area suggesting central necrosis and the presence of peripheral low attenuation suggesting a fatty hilum within an LN was considered a benign sign.

\section{Standard of reference}

Histopathological correlation was available in 16 patients and was used as the standard of reference. In the remaining 14 patients, the results of treatment change and clinical follow-up of at least 6 months including PET/CT, CT and MRI examinations served as reference. We classified as recurrence and/or metastasis the cases if, for example, (1) the present study revealed highly suspected recurrence without pathological evidence and the patient underwent chemotherapy resulting in a decrease or disappearance in size and/or FDG uptake in the follow-up study and (2) the follow-up study revealed tumor recurrence with $\mathrm{CT}$ and/or $\mathrm{PET} / \mathrm{CT}$ in a place where a tiny lesion without FDG uptake was imaged at the time of the initial (present) study (Fig. 2). 

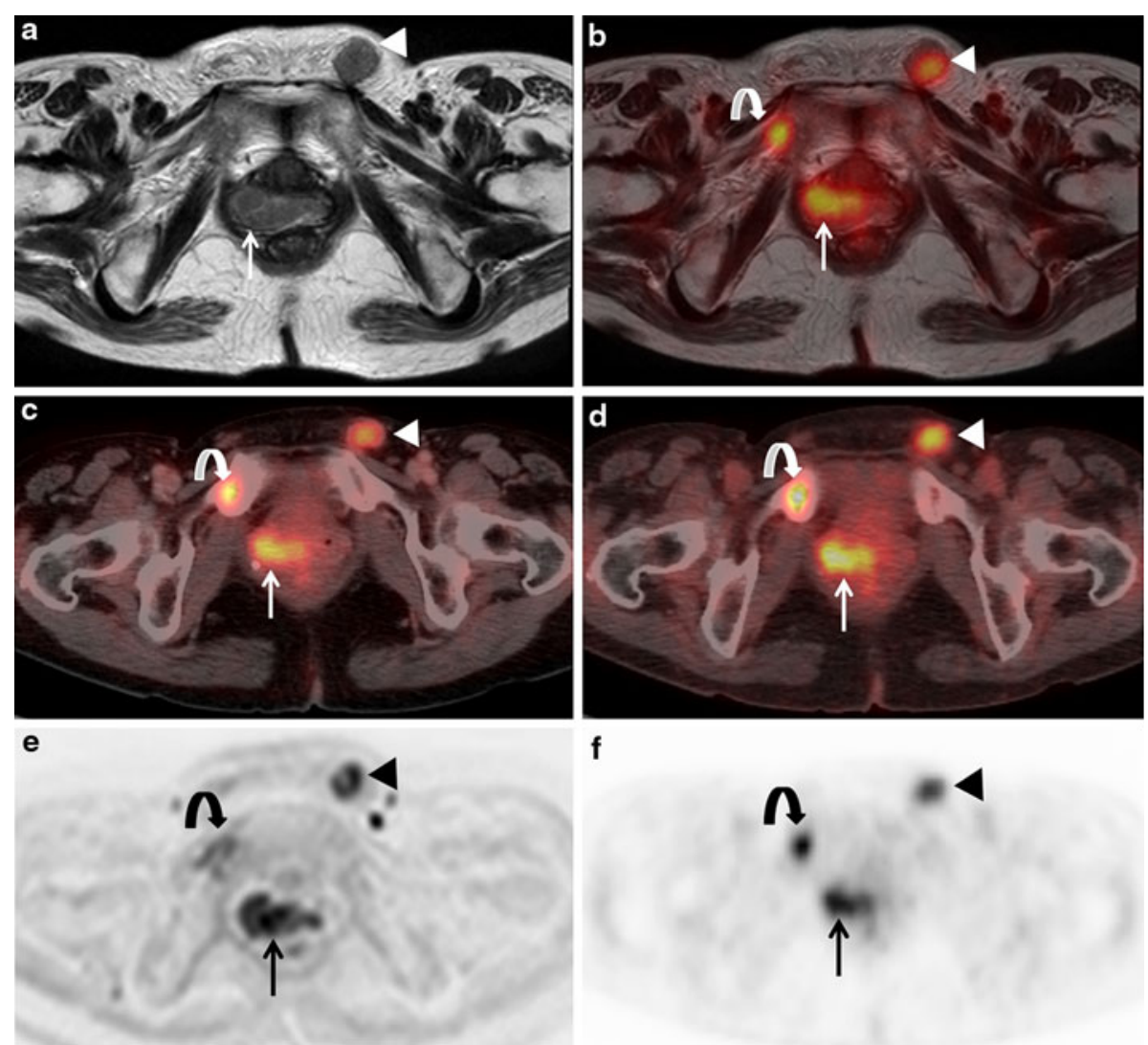

f

Fig. 1 A 63-year-old woman with local recurrence, bone and lymph node metastases after the surgery of uterine cervical cancer. a Axial T2-weighted MR image shows slightly hypointense masses at the right post-operative stump site (arrow) and left inguinal lymph node swelling (arrowhead) suspect of local recurrence and lymph node metastases. The abnormality of right pubis could not be detected. b Axial fused PET/MR image demonstrates intense FDG uptake of masses existing at right post-operative stump site (arrow), right pubis (curved arrow) and left inguinal region (arrowhead) suggesting local recurrence, bone and lymph node metastases. c Axial PET/ceCT image reveals abnormal FDG uptake at right post-operative stump site (arrow), right pubis (curved arrow), and left inguinal region (arrow head) suggesting local recurrence, bone and lymph node metastases.

\section{Statistical analysis}

The McNemar test was used to determine the statistical significance of differences in the sensitivity, specificity and accuracy as determined by PET/ceCT, PET/ldCT, MRI, and fused PET/MRI. Statistical analysis was performed with SAS software version 9.3 (SAS Institute, Cary, NC). A $p$ value less than 0.05 was considered to indicate a statistically significant difference.

\section{Result}

Documented positive locally recurrent disease, pelvic lymph node and bone metastases, and peritoneal dissemination were present in 16 of 30 patients $(53.3 \%), 8$ of 30 d Axial PET/ldCT image reveals abnormal FDG uptake at right postoperative stump site (arrow), right pubis (curved arrow), and left inguinal region (arrow head) suggesting local recurrence, bone and lymph node metastases. e Axial diffusion weighted MR image shows abnormal intensity of right post-operative stump site (arrow), right pubis (curved arrow), and left inguinal region (arrow head). f Axial PET image reveals abnormal FDG uptake at right post-operative stump site (arrow), right pubis (curved arrow), and left inguinal region (arrow head). All four protocols demonstrated true-positive findings of local recurrence and lymph node metastases. b Fused PET/ MRI, $\mathbf{c}$ PET/ceCT, and d PET/ldCT showed true true-positive finding of bone metastasis, however, a MRI showed false-negative finding

(26.7\%), 3 of $30(10.0 \%)$, and 5 of $30(16.7 \%)$, respectively. 16 patients had recurrence in only one of the four sites, five had recurrence in two sites, and two had recurrence in three sites. Seven patients proved to have no intrapelvic recurrent or metastatic lesions.

On per patient basis, the sensitivity, specificity and accuracy for the detection of intra-pelvic local recurrence were $87.5 \%$ (14/16), $100 \%(14 / 14)$ and $93.3 \%$ (28/30) for fused PET/MRI protocol, $87.5 \%$ (14/16), $100 \%$ (14/ 14) and $93.3 \%$ (28/30) for contrast-enhanced MRI protocol, $62.5 \%$ (10/16), $100 \%$ (14/14) and $80.0 \%$ (24/30) for PET/ceCT protocol, and $50.0 \%$ (8/16), $100 \%(14 / 14)$ and $73.3 \%$ (22/30) PET/ldCT protocol (Table 1). The sensitivity and accuracy of diagnosing local recurrence by fused PET/MRI were both significantly better than that of PET/ $\operatorname{ldCT}(p=0.041)$. There were no significant differences of 
Fig. 2 A 64-year-old woman with local recurrence after the surgery of endometrial cancer. a Axial post-contrast T1weighted fat-saturated MR image shows heterogeneously enhanced mass at the left postoperative stump site (arrow), suspect of local recurrence. b Axial fused PET/MR image demonstrates intense FDG uptake of the heterogeneously enhanced mass at the left postoperative stump site (arrow), suggesting local recurrence. c Axial PET/ceCT image reveals focal FDG uptake of the vagina (arrow), suspect of local recurrence. d Axial PET/ldCT image shows focal FDG uptake of the vagina (arrow). However, local recurrence was not suspected due to no abnormal findings by ldCT (not shown). e Axial diffusion weighted MR image shows abnormal intensity of the vagina (arrow). f Axial PET image reveals abnormal FDG uptake at the vagina (arrow). b Fused PET/MRI, a contrast-enhanced MRI, and b PET/ceCT demonstrated truepositive findings of local recurrence, however, d PET/ ldCT showed false-negative finding
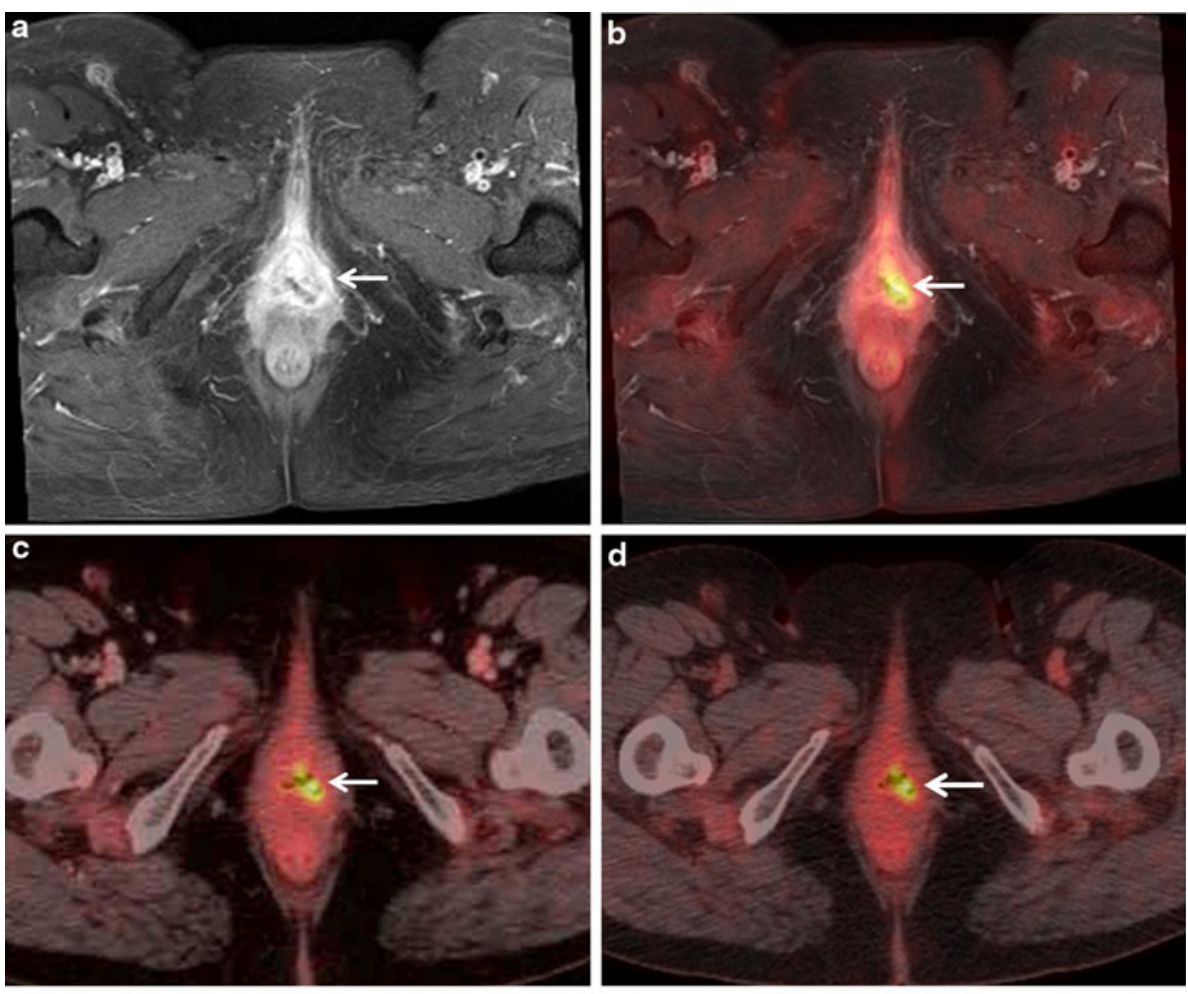

e sensitivity $(p=0.14)$ and accuracy $(p=0.14)$ between fused PET/MRI and PET/ceCT protocols. The patientbased sensitivity, specificity and accuracy for the detection of pelvic lymph node metastasis were $87.5 \%$ (7/8), $100 \%$ $(22 / 22)$ and $96.7 \%$ (29/30) for fused PET/MRI protocol, $62.5 \%(5 / 8), 100 \%(22 / 22)$ and $90.0 \%$ (27/30) for contrast-enhanced MRI protocol, $87.5 \%(7 / 8), 100 \%(22 / 22)$ and $96.7 \%$ (29/30) for PET/ceCT protocol and $87.5 \%(7 /$ 8), $95.5 \%(21 / 22)$ and $93.3 \%(28 / 30)$ for PET/ldCT protocol (Table 1). There were no significant differences of between fused PET/MRI and other protocols. The patientbased sensitivity, specificity and accuracy for the detection of pelvic bone metastasis were $100 \%$ (3/3), $100 \%(27 / 27)$ and $100 \%$ (30/30) for fused PET/MRI protocol, $66.7 \%$ (2/ $3), 100 \%(27 / 27)$ and $96.7 \%(29 / 30)$ for contrastenhanced MRI protocol, $66.7 \%$ (2/3), $100 \%$ (27/27) and $96.7 \%$ (29/30) for PET/ceCT protocol and $66.7 \%(2 / 3)$, $100 \%(27 / 27)$ and $96.7 \%$ (29/30) for PET/ldCT protocol
(Table 1). There were no significant differences of between fused PET/MRI and other protocols. The patient-based sensitivity, specificity and accuracy for the detection of peritoneal dissemination were $80.0 \%(4 / 5), 100 \%(25 / 25)$ and $96.7 \%$ (29/30) for fused PET/MRI protocol, $60.0 \%$ $(3 / 5), 100 \%(25 / 25)$ and $93.3 \%(28 / 30)$ for contrastenhanced MRI protocol, $80.0 \%(4 / 5), 100 \%(25 / 25)$ and $96.7 \%$ (29/30) for PET/ceCT protocol and $60.0 \%(3 / 5)$, $96.0 \%(24 / 25)$ and $90.0 \%$ (27/30) for PET/ldCT protocol (Table 1). There were no significant differences of between fused PET/MRI and other protocols.

The patient-based sensitivity, specificity and accuracy for the detection of intra-pelvic recurrence/metastasis were $91.3 \%$ (21/23), $100 \%$ (7/7) and $93.3 \%$ (28/30) for fused PET/MRI protocol, $82.6 \%$ (19/23), $100 \%(7 / 7)$ and $86.7 \%$ (26/30) for contrast-enhanced MRI protocol, $82.6 \%$ (19/23), $100 \%$ (7/7) and $86.7 \%$ (26/30) for PET/ ceCT protocol and $78.3 \%(18 / 23), 85.7 \%(6 / 7)$ and 
Table 1 Comparison of fused PET/MR, PET/CT and MRI for diagnosing intra-pelvic recurrence on a per patient basis

\begin{tabular}{|c|c|c|c|c|}
\hline & $\begin{array}{l}\text { Fused PET/ } \\
\text { MR }(\%)\end{array}$ & $\begin{array}{l}\text { MRI } \\
(\%)\end{array}$ & $\begin{array}{l}\mathrm{PET} / \mathrm{ceCT} \\
(\%)\end{array}$ & $\begin{array}{l}\mathrm{PET} / \mathrm{ldCT} \\
(\%)\end{array}$ \\
\hline \multicolumn{5}{|c|}{ Local recurrence } \\
\hline Sensitivity & $87.5(14 / 16)^{*}$ & $87.5(14 / 16)$ & $62.5(10 / 16)$ & $50.0(8 / 16)^{*}$ \\
\hline Specificity & $100(14 / 14)$ & $100(14 / 14)$ & $100(14 / 14)$ & $100(14 / 14)$ \\
\hline Accuracy & $93.3(28 / 30)^{*}$ & $93.3(28 / 30)$ & $80.0(24 / 30)$ & $73.3(22 / 30)^{*}$ \\
\hline \multicolumn{5}{|c|}{ Pelvic lymph node metastasis } \\
\hline Sensitivity & $87.5(7 / 8)$ & $62.5(5 / 8)$ & $87.5(7 / 8)$ & $87.5(7 / 8)$ \\
\hline Specificity & $100(22 / 22)$ & $100(22 / 22)$ & $100(22 / 22)$ & $95.5(21 / 22)$ \\
\hline Accuracy & $96.7(29 / 30)$ & $90.0(27 / 30)$ & $96.7(29 / 30)$ & $93.3(28 / 30)$ \\
\hline \multicolumn{5}{|c|}{ Bone metastasis } \\
\hline Sensitivity & $100(3 / 3)$ & $66.7(2 / 3)$ & $66.7(2 / 3)$ & $66.7(2 / 3)$ \\
\hline Specificity & $100(27 / 27)$ & $100(27 / 27)$ & $100(27 / 27)$ & $100(27 / 27)$ \\
\hline Accuracy & $100(30 / 30)$ & $96.7(29 / 30)$ & $96.7(29 / 30)$ & $96.7(29 / 30)$ \\
\hline \multicolumn{5}{|c|}{ Peritoneal dissemination } \\
\hline Sensitivity & $80.0(4 / 5)$ & $60.0(3 / 5)$ & $80.0(4 / 5)$ & $60.0(3 / 5)$ \\
\hline Specificity & $100(25 / 25)$ & $100(25 / 25)$ & $100(25 / 25)$ & $96.0(24 / 25)$ \\
\hline Accuracy & $96.7(29 / 30)$ & $93.3(28 / 30)$ & $96.7(29 / 30)$ & $90.0(27 / 30)$ \\
\hline
\end{tabular}

PET/ceCT PET/full-dose contrast-enhanced CT, PET/ldCT PET/low-dose nonenhanced CT

* The sensitivity and accuracy of diagnosing local recurrence between fused PET/MR and PET/ldCT were statistically different $(p=0.041)$

$80.0 \%$ (24/30) for PET/ldCT protocol. Among four modalities, fused PET/MRI showed the highest sensitivity and accuracy. However, the difference did not reach significant difference about the sensitivity $(p=0.48)$, specificity $(p=1)$, and accuracy $(p=0.48)$.

\section{Discussion}

To our knowledge, this is the first reported study to have investigated the validity of retrospectively fused PET/MRI obtained from different scanners for assessing intra-pelvic recurrence of gynecological cancers and to compare the diagnostic accuracy of fused PET/MRI with that of contrast-enhanced PET/CT using ${ }^{18} \mathrm{~F}-\mathrm{FDG}$ and pelvic contrastenhanced MRI. Among three modalities, fused PET/MRI showed the highest sensitivity and accuracy. This result may be because of excellent spatial resolution and softtissue contrast of MRI and functional method based on the increased glucose metabolism of cancer cells, regardless of node size of ${ }^{18}$ F-FDG PET/CT [10].

There has been only one study to evaluate the additional diagnostic value of fused PET/MRI in the detection of pelvic and para-aortic metastatic lymph nodes in uterine cervical cancer patients. Kim et al. [3] demonstrated that in a study of seventy nine patients with FIGO stage IB-IVA cervical cancer, region-based sensitivity, specificity and area under the receiver operating characteristic (ROC) of
Fig. 3 A 38-year-old woman with local recurrence and peritoneal dissemination after the surgery of ovarian cancer. a Axial T2weighted MR image shows a hypointense mass between the bladder and the rectum (arrow), suspect of local recurrence. b Axial fused PET/MR image demonstrates intense FDG uptake of the hyperintense mass between the bladder and the rectum (arrow), suggesting local recurrence. c Axial PET/ceCT image shows focal FDG uptake in the middle of the pelvis (arrow), suspect of bowel physiological uptake. d Axial PET/ldCT image shows focal FDG uptake in the middle of the pelvis (arrow), suspect of bowel physiological uptake. e Axial diffusion weighted MR image shows abnormal intensity in the location of pointed by a T2-weighted MR image (arrow). f Axial PET image reveals abnormal FDG uptake in the pelvis (arrow). g Axial T2-weighted MR image shows a hyperintense mass existing on the intestinal wall (arrow), however this mass could not be detected by readers. h Axial fused PET/MR image demonstrates intense FDG uptake of a hyperintense mass existing on the intestinal wall (arrow), suggesting peritoneal dissemination. i Axial PET/ceCT image reveals intense FDG uptake of an enhanced mass existing on the intestinal wall (arrow), suggesting peritoneal dissemination. j Axial PET/ldCT image shows focal FDG uptake on the intestinal wall (arrow), suspect of bowel physiological uptake. $\mathbf{k}$ Axial diffusion weighted MR image shows abnormal intensity in the location of pointed by $\mathbf{f} \mathrm{T} 2$-weighted MR image (arrow). I Axial PET image reveals abnormal FDG uptake in the left lower abdomen (arrow). b PET/MRI and a MRI showed true true-positive finding of local recurrence, however $\mathbf{c}, \mathbf{d}$ PET/CT showed false-negative finding. b PET/MRI and i PET/ceCT showed true true-positive findings of peritoneal dissemination, however, f MRI and $\mathbf{j}$ PET/ldCT showed false-negative finding

detecting pelvic and para-aortic lymph node metastases were $54.2 \%, 92.7 \%$ and 0.735 for fused PET/MRI, and those figures were $44.1 \%, 93.9 \%$ and 0.690 for PET/CT. They also reported a significant difference of ROC analysis $(p=0.045)$; however, we suppose the inferiority of PET/ CT result may arise because contrast-enhanced medium was not used in CT scan of PET/CT study. In our series of PET/CT with contrast-enhanced medium, the difference was not observed between fused PET/MRI and PET/ceCT. ${ }^{18} \mathrm{~F}$-FDG PET is a functional method based on the increased glucose metabolism of cancer cells, regardless of node size, and PET/CT can often detect tiny metastatic LN from 5 to $9 \mathrm{~mm}$ in size which could not be diagnosed by MRI or CT [12]. In general, the sensitivity of diagnosing lymph node metastases by PET/CT is higher than that of CT or MRI.

Antonsen et al. [13] compared the primary tumor and nodal staging of endometrial cancer between preoperative contrast-enhanced PET/CT using ${ }^{18} \mathrm{~F}$-FDG and contrastenhanced MRI. They demonstrated that in a study of 318 patients with FIGO stage IA-IVB endometrial cancer, $\mathrm{PET} / \mathrm{ceCT}$ and contrast-enhanced MRI were equal in predicting $\geq 50 \%$ myometrial invasion, uterine serosa, cervical involvement and lymph node metastases. The sensitivity and specificity of detecting $\geq 50 \%$ myometrial invasion were 93 and $49 \%$ for PET/CT and 87 and $57 \%$ for MRI. The sensitivity and specificity of detecting uterine serosa invasion were 75 and $99 \%$ for PET/CT and 67 and $96 \%$ for MRI. The sensitivity and specificity of cervical 

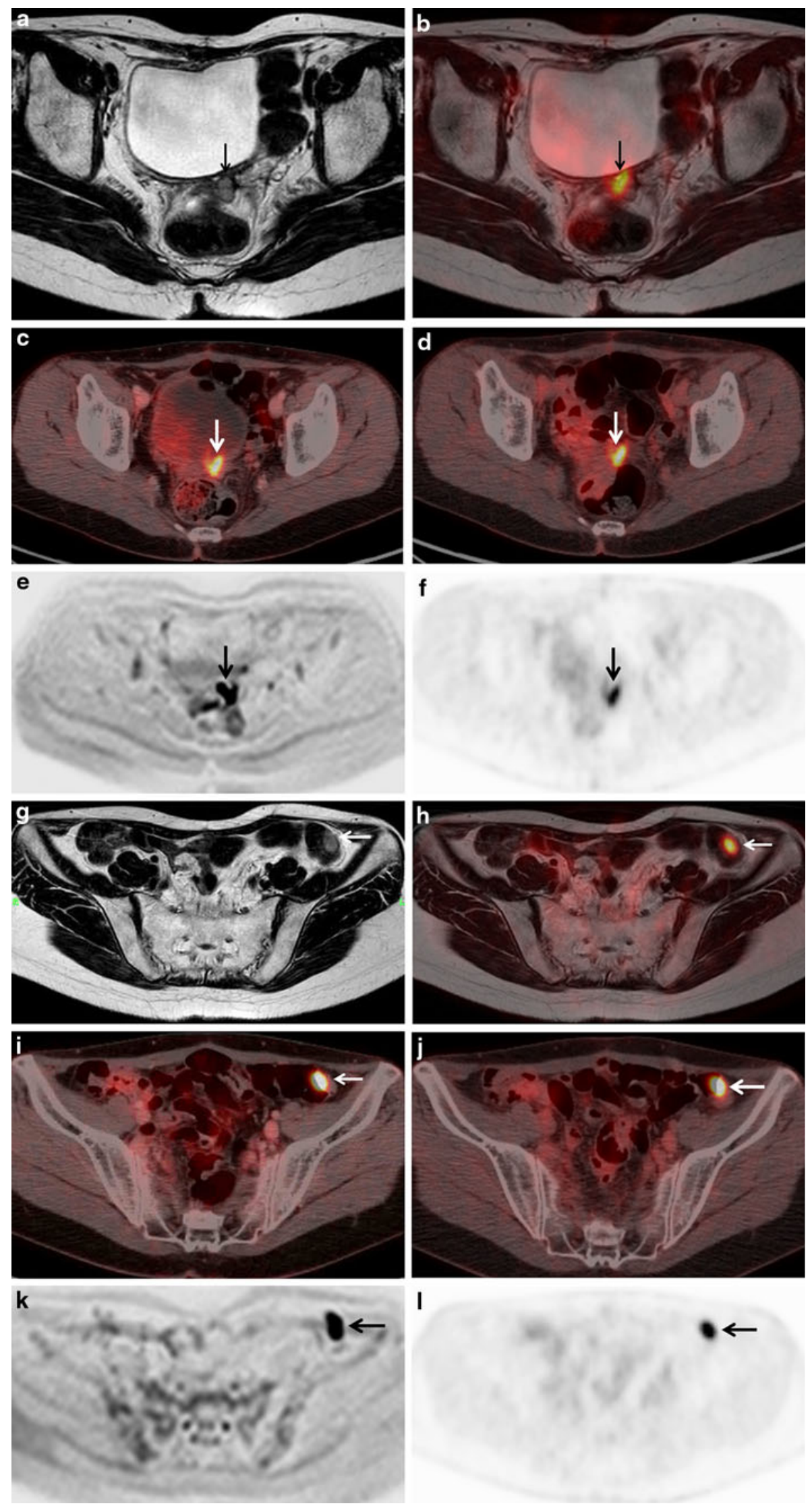

$\bullet \leftarrow$ 
involvement were 43 and $94 \%$ for PET/CT and 33 and $95 \%$ for MRI. The sensitivity and specificity of detecting para-aortic and pelvic lymph node metastases were 74 and $93 \%$ for PET/CT and 59 and $93 \%$ for MRI.

In this study, PET/MR images were generated from PET and MR images obtained from different scanners and PET and MR images were merged and registered manually on a workstation. A drawback of the PET/MRI fusion images is misregistration due to the movement of organs between the PET and MRI studies. Pelvic organs may show little respiratory movement, but urinary volume and location of gas in the intestine, colon, and rectum may affect the location of surrounding organs. Inline PET/MRI technology may be expected to minimize these drawbacks of the PET/MRI fusion strategy and generate better image quality for fusion images (Fig. 3).

This study has several limitations. First, this was a retrospective study. PET/CT was not performed in every case of restaging gynecological cancer during the study period. The application of PET/CT to only selected cases might cause bias and influence the study results. The size of patient sample was relatively small. Further prospective and larger studies are needed. Second, the ideal gold standard for any analysis would be histological confirmation of the findings. However, clinical follow-up is a valid way to evaluate diagnostic accuracy and response to therapy, and it would have been unethical to investigate all imaging-detected lesions using invasive procedures. Positive findings are easy to confirm, but negative findings only mean that it is not possible to acquire positive findings during the follow-up period, making it uncertain whether the findings are truly negative. Therefore, sensitivity in this series may have been overestimated. Third, pelvic MR images but not whole-body MR images were used for PET/ MRI fusion images. Therefore, intra-pelvic status was evaluated in our series. For a comparative study of diagnostic ability for distant metastasis, whole-body MRI is needed for the fusion images.

\section{Conclusion}

Fused PET/MRI, combining the individual advantages of MRI and PET, is a very useful modality for assessing intrapelvic recurrence of gynecological cancers. The combination of whole-body PET and MRI into single scanner is a very promising diagnostic modality for oncological imaging due to the missing radiation exposure and the high soft tissue resolution of MRI in contrast to CT.

Acknowledgments We wish to thank Takashi Okunaga RT and Hajime Aoki RT (Radiology Division, Kobe University Hospital, Kobe, Japan) and for their excellent technical assistance and generous support. We also wish to express special thanks to Hirofumi Kawakami RT (GE healthcare) for his outstanding technical assistance.

Conflict of interest We declare no financial support or relationship that may pose conflict of interest.

\section{References}

1. Townsend DW. Dual-modality imaging: combining anatomy and function. J Nucl Med. 2008;49:938-55.

2. von Schulthess GK, Steinert HC, Hany TF. Integrated PET/CT: current applications and future directions. Radiology. 2006;238: 405-22.

3. Kim SK, Choi HJ, Park SY, Lee HY, Seo SS, Yoo CW, et al. Additional value of MR/PET fusion compared with PET/CT in the detection of lymph node metastases in cervical cancer patients. Eur J Cancer. 2009;45:2103-9.

4. Nakajo K, Tatsumi M, Inoue A, Isohashi K, Higuchi I, Kato H, et al. Diagnostic performance of fluorodeoxyglucose positron emission tomography/magnetic resonance imaging fusion images of gynecological malignant tumors: comparison with positron emission tomography/computed tomography. Jpn J Radiol. 2010;28:95-100.

5. Kitajima K, Suenaga Y, Ueno Y, Kanda T, Maeda T, Takahashi $\mathrm{S}$, et al. Value of fusion of PET and MRI for staging of endometrial cancer: comparison with ${ }^{18} \mathrm{~F}-\mathrm{FDG}$ contrast-enhanced PET/CT and dynamic contrast-enhanced pelvic MRI. Eur J Radiol. 2013;82:1672-6.

6. Kanda T, Kitajima K, Suenaga Y, Konishi J, Sasaki R, Morimoto $\mathrm{K}$, et al. Value of retrospective image fusion of 18F-FDG PET and MRI for preoperative staging of head and neck cancer: comparison with PET/CT and contrast-enhanced neck MRI. Eur. J. Radiol. 2013 (Epub ahead of print).

7. Low RN, Duggan B, Barone RM, Saleh F, Thomas Song SY. Treated ovarian cancer: MR imaging, laparotomy reassessment, and serum CA-125 values compared with clinical outcome at 1 year. Radiology. 2005;235:918-26.

8. Jeong YY, Kong HK, Chung TW, Seo JJ, Park JG. Uterine cervical carcinoma after therapy: CT and MR imaging findings. Radiographics. 2003;23:968-81.

9. Nishie A, Stolpen AH, Obuchi M, Kuehn DM, Dagit A, Andresen K. Evaluation of locally recurrent pelvic malignancy: performance of T2- and diffusion-weighted MRI with image fusion. J Magn Reson Imaging. 2008;28:705-13.

10. Kitajima K, Murakami K, Yamasaki E, Domeki Y, Kaji Y, Fukasawa I, et al. Performance of integrated FDG-PET/contrastenhanced CT in the diagnosis of recurrent ovarian cancer: comparison with integrated FDG-PET/non-contrast-enhanced CT and enhanced CT. Eur J Nucl Med Mol Imaging. 2008;35:1439-48.

11. Kitajima K, Murakami K, Yamasaki E, Domeki Y, Kaji Y, Morita S, et al. Performance of integrated FDG-PET/contrastenhanced CT in the diagnosis of recurrent uterine cancer: comparison with PET and enhanced CT. Eur J Nucl Med Mol Imaging. 2009;36:362-72.

12. Kitajima K, Murakami K, Yamasaki E, Kaji Y, Sugimura K. Accuracy of integrated FDG-PET/contrast-enhanced CT in detecting pelvic and paraaortic lymph node metastasis in patients with uterine cancer. Eur Radiol. 2009;19:1529-36.

13. Antosen SL, Jensen LN, Loft A, Berthelsen AK, Costa J, Tabor A, et al. MRI, PET/CT and ultrasound in the preoperative staging of endometrial cancer-a multicenter prospective comparative study. Gynecol Oncol. 2013;128:300-8. 